\title{
SIMPLICITY OF THE AUTOMORPHISM GROUPS OF GENERALISED METRIC SPACES
}

\author{
DAVID M. EVANS, JAN HUBIČKA, MATĚJ KONEČNÝ, YIBEI LI, \\ AND MARTIN ZIEGLER
}

\begin{abstract}
Tent and Ziegler proved that the automorphism group of the Urysohn sphere is simple and that the automorphism group of the Urysohn space is simple modulo bounded automorphisms. A key component of their proof is the definition of a stationary independence relation (SIR). In this paper we prove that the existence of a SIR satisfying some extra axioms is enough to prove simplicity of the automorphism group of a countable structure. The extra axioms are chosen with applications in mind, namely homogeneous structures which admit a "metric-like amalgamation", for example all primitive 3-constrained metrically homogeneous graphs of finite diameter from Cherlin's list.
\end{abstract}

\section{INTRODUCTION}

In 2011, Macpherson and Tent [MT11] proved that the automorphism groups of Fraïssé limits of free amalgamation classes are simple. This was followed by two papers of Tent and Ziegler [TZ13b, TZ13a] where they prove that the isometry group of the Urysohn space (the unique complete separable homogeneous metric space universal for all finite metric spaces) modulo bounded isometries (i.e. isometries $f$ with a finite bound on the distance between $x$ and $f(x))$ is simple and that the isometry group of the Urysohn sphere is simple. Later, Evans, Ghadernezhad and Tent [EGT16] proved simplicity for automorphism groups of some Hrushovski constructions, and Li [Li18] proved simplicity for the structures from Cherlin's list of 26 primitive triangle-constrained homogeneous structures with 4 binary symmetric relations (see appendix of [Che98]).

More recently, Tent and Ziegler's method was generalised to asymmetric structures. Li [Li19] proved that the automorphism groups of some of Cherlin's asymmetric structures in the appendix of [Che98] are simple. The same result for nontrivial linearly ordered free homogeneous structures has been proved independently by Calderoni, Kwiatkowska and Tent [CKT20] and Li [Li20]. Also in [Li20], simplicity was proved for the automorphism groups of the universal $n$-linear orders for $n \geq 2$. Another recent example where (non-stationary) independence relations have been used to prove strong results about automorphism groups of structures is a paper by Kaplan and Simon [KS19].

In this paper, we adapt the methods of Tent and Ziegler and prove the following theorem (definitions and examples will be given in the upcoming paragraphs).

Theorem 1.1. Let $\mathbb{F}$ be a transitive countable relational structure with a bounded 1supported metric-like stationary independence relation $\downarrow$. Then Aut $(\mathbb{F})$ is simple.

Jan Hubička and Matěj Konečný are supported by project 18-13685Y of the Czech Science Foundation (GAČR) and by a project that has received funding from the European Research Council (ERC) under the European Union's Horizon 2020 research and innovation programme (grant agreement No 810115). Matěj Konečný is supported by the Charles University project GA UK No 378119. Yibei Li is supported by President's scholarship from Imperial College. Jan Hubička is supported by the Center for Foundations of Modern Computer Science (Charles University project UNCE/SCI/004). 
As direct corollaries of Theorem 1.1, we get the following two more concrete results, for which the definitions will be given in Section 4 .

Theorem 1.2. Let $\mathfrak{M}=(M, \oplus, \preceq)$ be a finite archimedean partially ordered commutative semigroup with at least two elements and let $\mathbb{F}$ be a homogeneous $\mathfrak{M}$-metric space which realises all distances. Assume that $\mathbb{F}$ admits an $\mathfrak{M}$-shortest path independence relation $\downarrow$ and that $\downarrow$ is a 1-supported SIR. Then $\operatorname{Aut}(\mathbb{F})$ is simple.

Theorem 1.3. If $G$ is a countably infinite metrically homogeneous graph which corresponds to one of the primitive 3-constrained finite-diameter classes from Cherlin's catalogue [Che11], then $\operatorname{Aut}(G)$ is simple.

1.1. Stationary independence relations. The notion of stationary independence relations (Definition 1.4) was developed by Tent and Ziegler in their paper on the Urysohn space [TZ13b]. It has several generalisations (e.g. for structures with closures [EGT16]), but for our purposes the original variant suffices.

Let $\mathbb{F}$ be a relational structure and let $A, B \subseteq \mathbb{F}$ be finite subsets. We will identify them with the substructures induced by $\overline{\mathbb{F}}$ on $A$ and $B$ respectively and by $A B$ we will denote the union $A \cup B$ (and hence also the substructure induced by $\mathbb{F}$ on $A B)$. If the set $A=\{a\}$ is singleton, we may write $a$ instead of $\{a\}$. Uppercase letters will denote sets while lowercase will denote the elements of the structure, which we call vertices owing to the combinatorial background of part of the authors. As is usual in this area, if $A \subseteq \mathbb{F}$, we sometimes assume that it has some implicit enumeration. This is clear from the context and should not cause any confusion.

Let $A, X \subseteq \mathbb{F}$. By the type of $A$ over $X$ (denoted by $\operatorname{tp}(A / X)$ ) we mean the orbit of $A$ under the action of the stabilizer subgroup of $\operatorname{Aut}(\mathbb{F})$ with respect to $X$. If $p=\operatorname{tp}(A / X)$, we say that $B \subseteq \mathbb{F}$ realises $p$ (and denote it as $B \models p$ ) if $B$ lies in $p$, in other words, if there is an automorphism of $\mathbb{F}$ fixing $X$ pointwise which maps $A$ to $B$. To simplify the notation, we write $\operatorname{tp}(A)$ for $\operatorname{tp}(A / \emptyset)$. Our types correspond to realised types in a (strongly) homogeneous structure in the standard model-theoretic terminology. In fact, we may assume that the language is chosen so that $\mathbb{F}$ is homogeneous, that is, partial automorphisms between finite substructures of $\mathbb{F}$ extend to automorphisms.

Definition 1.4 (Stationary Independence Relation). Let $\mathbb{F}$ be a relational structure. A ternary relation $\downarrow$ on finite subsets of $\mathbb{F}$ is called a stationary independence relation ( $S I R$, with $A \downarrow_{C} B$ being pronounced " $A$ is independent from $B$ over $C$ ") if the following conditions are satisfied:

SIR1 (Invariance). The independence of finite subsets of $\mathbb{F}$ only depends on their type. In particular, for every automorphism $f$ of $\mathbb{F}$, we have $A \downarrow_{C} B$ if and only if $f(A) \downarrow_{f(C)} f(B)$.

SIR2 (Symmetry). If $A \downarrow_{C} B$, then $B \downarrow_{C} A$.

SIR3 (Monotonicity). If $A \downarrow_{C} B D$, then $A \downarrow_{C} B$ and $A \downarrow_{B C} D$.

SIR4 (Existence). For every $A, B$ and $C$ in $\mathbb{F}$, there is some $A^{\prime} \models \operatorname{tp}(A / C)$ with $A^{\prime} \mathfrak{L}_{C} B$.

SIR5 (Transitivity) If $A \downarrow_{C} B$ and $A \downarrow_{B C} B^{\prime}$, then $A \downarrow_{C} B^{\prime}$.

SIR6 (Stationarity) If $A$ and $A^{\prime}$ have the same type over $C$ and are both independent over $C$ from some set $B$ then they also have the same type over $B C$.

Note that by an observation of Baudisch [Bau16], these axioms are redundant as Monotonicity can be derived from the rest of them. Stationary independence relations correspond to "canonical amalgamations" by putting $A \downarrow_{C} B$ if and only 
if the canonical amalgamation of $A C$ and $B C$ over $C$ is isomorphic to $A B C$. The notion of canonical amalgamations can be formalised, see $\left[\mathrm{ABWH}^{+} 17 \mathrm{c}\right]$.

To make our proofs shorter, we will sometimes use Symmetry, Monotonicity and Existence implicitly. The following observation which follows from Invariance will be useful later.

Observation 1.5. If $\mathbb{F}$ is a relational structure, $\downarrow$ a SIR on $\mathbb{F}$ and $A \downarrow_{C} B$, then $A \downarrow_{C} B C$.

Definition 1.6 ( $k$-supported SIR). Let $k$ be a positive integer. We say that a SIR $\downarrow$ is $k$-supported if for every $a, b, C$ such that $a \downarrow_{C} b$ there is $C^{\prime} \subseteq C$ such that $\left|C^{\prime}\right| \leq k$ and $a \downarrow_{C^{\prime}} b$.

Observation 1.7. For $k=1, k$-supportedness is equivalent to:

(1-supportedness) If $a \downarrow_{C} b$ and $C=C_{1} \cup C_{2}$ then $a \downarrow_{C_{1}} b$ or $a \downarrow_{C_{2}} b$.

We say that a structure $\mathbb{F}$ is transitive if $\operatorname{tp}(a)=\operatorname{tp}(b)$ for every $a, b \in \mathbb{F}$.

Definition 1.8 (Metric-like SIR). Let $\mathbb{F}$ be a relational structure with a SIR $\downarrow$. We say that $\downarrow$ is metric-like if the following conditions are satisfied:

(1) If $a \notin A$, then $a \mathbb{Z}_{A} a$.

(2) For every $a \in \mathbb{F}$ there is $b \in \mathbb{F}$ such that $a \neq b$ and $a \mathbb{Z}_{\emptyset} b$.

(3) (Perfect triviality) If $A \downarrow_{C} B$ and $C \subseteq C^{\prime}$ then $A \downarrow_{C^{\prime}} B$.

Lemma 1.9. Let $\mathbb{F}$ be a relational structure with a SIR $\downarrow$ which satisfies Perfect triviality. Then $\downarrow$ satisfies

(1) (Metricity) If $A \downarrow_{C_{1} C_{2}} B$ and $C_{1} \downarrow_{D} B$ then $A \downarrow_{C_{2} D} B$.

(2) (Triviality) If $A \downarrow_{B} C$ and $A \downarrow_{B} D$ then $A \downarrow_{B} C D$.

Proof. First assume that $A \downarrow_{C_{1} C_{2}} B$ and $C_{1} \downarrow_{D} B$. By Perfect triviality, $C_{1} \downarrow_{C_{2} D} B$ and $A \downarrow_{C_{1} C_{2} D} B$. Using Transitivity it follows that $A \downarrow_{C_{2} D} B$, which proves Metricity.

Now assume that $A \downarrow_{B} C$ and $A \downarrow_{B} D$. By Perfect triviality we get $A \downarrow_{B C} D$ and by Observation 1.5 and Monotonicity it then follows that that $A \downarrow_{B C} C D$. Using Transitivity together with $A \downarrow_{B} C$ then implies $A \downarrow_{B} C D$.

In fact, Metricity is equivalent to Perfect triviality if $\downarrow$ is a SIR. The following is a simple corollary of Triviality which will be useful later.

Corollary 1.10. If $a \downarrow_{\emptyset} x$ for every $x \in X$, then $a \downarrow_{\emptyset} X$.

Definition 1.11 (Geodesic sequence). Let $\mathbb{F}$ be a relational structure with a SIR $\downarrow$. We say that a sequence $a_{1}, \ldots, a_{n} \in \mathbb{F}$ of pairwise distinct vertices of $\mathbb{F}$ is geodesic if for every $1 \leq i<j<k \leq n$ it holds that $a_{i} \downarrow_{a_{j}} a_{k}$.

Definition 1.12. Let $\mathbb{F}$ be a relational structure with a SIR $\downarrow$. We say that $\downarrow$ is bounded if it satisfies

(Boundedness) There exists an integer $k_{0}$ such that if $a_{0}, \ldots, a_{k}$ is a geodesic sequence with $k \geq k_{0}$, then $a_{0} \downarrow_{\emptyset} a_{k}$.

We denote the smallest such $k_{0}$ by $\|\downarrow\|$.

The reader is encouraged to have the following examples in mind when reading this paper. 
Example 1. Let $\mathbb{F}$ be the Fraïssé limit of all finite metric spaces using only distances $\{0,1, \ldots, n\}$ for some fixed $n \geq 2$ (clearly, one can view a metric space as a relational structure by introducing a binary relation for every distance). Define $\downarrow$ on $\mathbb{F}$ by putting $A \downarrow_{C} B$ if and only if for every $a \in A$ and every $b \in B$ it holds that $d(a, b)=\min (\{n\} \cup\{d(a, c)+d(b, c): c \in C\})$. It is straightforward to check that $\downarrow$ is a bounded 1-supported metric-like SIR with $\|\downarrow\|=n$.

For the Urysohn sphere, the only axiom which we do not have at hand is, paradoxically, Boundedness.

Example 2. Let $\mathbb{U}_{1}$ be the Urysohn sphere, that is, the unique homogeneous separable complete metric space with distances from $[0,1]$ which is universal for all finite metric spaces with distances from $[0,1]$. We will denote its metric by $d$. Define the relation $\downarrow$ on finite subsets of $\mathbb{U}_{1}$ by putting $A \downarrow_{C} B$ if and only if for every $a \in A$ and every $b \in B$ it holds that $d(a, b)=\min (\{1\} \cup\{d(a, c)+d(b, c): c \in$ $C\})$. One can check that $\downarrow$ is a 1-supported metric-like SIR, but does not satisfy Boundedness, as for every $k$ one can find a geodesic sequence with $k+1$ vertices such that the distance of every consecutive pair of them is smaller that $\frac{1}{k}$.

Example 3 ( $k$-supported metric-like SIR). Let $k \geq 1$ and $n \geq 3$ be integers. Put $S=\{1, \ldots, n\}^{k} \cup\{0\}^{k}$, let $A$ be a set and let $d: A^{2} \rightarrow S$ be a function. Let $\preceq$ be the product order on $S$ (i.e. $\left(a_{1}, \ldots, a_{k}\right) \preceq\left(b_{1}, \ldots, b_{k}\right)$ if and only if $a_{i} \leq b_{i}$ for every $1 \leq i \leq k)$ and let $\oplus$ be the component-wise addition on $S$ capped at $n$ (i.e. $\left(a_{1}, \ldots, a_{k}\right) \oplus\left(b_{1}, \ldots, b_{k}\right)=\left(c_{1}, \ldots, c_{k}\right)$, where $c_{i}=\min \left(n, a_{i}+b_{i}\right)$ for every $1 \leq i \leq k)$.

We say that $(A, d)$ is an $[n]^{k}$-metric space if the following holds for every $x, y, z \in$ A:

(1) $d(x, y)=d(y, x)$,

(2) $d(x, y)=(0, \ldots, 0)$ if and only if $x=y$,

(3) $d(x, z) \preceq d(x, y) \oplus d(y, z)$.

One can verify that the class of all finite $[n]^{k}$-metric spaces is a Fraïssé class. Consider the structure $\mathbb{M}_{k}=\left(M_{k}, d\right)$, which is the Fraïssé limit of the class of all $[n]^{k}$-metric spaces, and define $\downarrow$ on $\mathbb{M}_{k}$ by putting $A \downarrow_{C} B$ if and only if for every $a \in A$ and every $b \in B$ it holds that $d(a, b)=\inf _{\preceq}\{d(a, c) \oplus d(c, b): c \in C\}$. As $\preceq$ has a maximum, the infimum of the empty set is $(n, \ldots, n)$.

It is easy to verify that $\downarrow$ is a bounded metric-like SIR. Moreover, it is $k$ supported, but not $k^{\prime}$-supported for any $k^{\prime}<k$, which is witnessed by vertices $a, b, c_{1}, \ldots, c_{k} \in \mathbb{M}_{k}$ such that $a \downarrow_{\left\{c_{1}, \ldots, c_{k}\right\}} b, d\left(a, c_{i}\right)=(1, \ldots, 1)$ for every $i$ and $d\left(b, c_{i}\right)$ is equal to 1 on the $i$-th coordinate and equal to 2 everywhere else.

\section{Geodesic SEQuences}

In this section we prove some auxiliary results about geodesic sequences which will be used later. Fix a transitive relational structure $\mathbb{F}$ with a metric-like SIR $\downarrow$.

Lemma 2.1. Let $a_{1}, \ldots, a_{n}$ be a geodesic sequence of vertices of $\mathbb{F}$ and let $b \in$ $\mathbb{F} \backslash\left\{a_{n}\right\}$. Then there is $a_{n+1} \models \operatorname{tp}\left(b / a_{n}\right)$ such that $a_{1}, \ldots, a_{n+1}$ is a geodesic sequence.

Proof. Using Existence, pick $a_{n+1} \models \operatorname{tp}\left(b / a_{n}\right)$ such that $a_{1} \cdots a_{n-1} \downarrow_{a_{n}} a_{n+1}$. Consider any $1 \leq i<j \leq n-1$. By Monotonicity, $a_{i} \downarrow_{a_{n}} a_{n+1}$ and hence, by Perfect triviality, $a_{i} \downarrow_{a_{j} a_{n}} a_{n+1}$. Since $a_{1}, \ldots, a_{n}$ is a geodesic sequence, we know that $a_{i} \downarrow_{a_{j}} a_{n}$. Transitivity now implies that $a_{i} \downarrow_{a_{j}} a_{n+1}$ and hence $a_{1}, \ldots, a_{n+1}$ is a geodesic sequence. 
Lemma 2.2. Let $a, b, c \in \mathbb{F}$ be distinct such that $a \downarrow_{\emptyset} b$. There is a geodesic sequence $a_{0}, a_{1}, \ldots, a_{n} \in \mathbb{F}$ satisfying the following:

(1) $a=a_{0}$ and $b=a_{n}$, and

(2) for every $0 \leq i \leq n-1$ it holds that $\operatorname{tp}\left(a_{i} a_{i+1}\right)=\operatorname{tp}(a c)$,

(3) $n=\|\downarrow\|$.

Proof. First observe that since all vertices have the same type, for every $v \in \mathbb{F}$ there is $v^{\prime} \in \mathbb{F}$ such that $\operatorname{tp}\left(v v^{\prime}\right)=\operatorname{tp}(a c)$. Put $n=\|\downarrow\|$ and use Lemma 2.1 repeatedly to obtain a geodesic sequence $a, x_{1}, \ldots, x_{n}$ such that all consecutive pairs of vertices have the type $\operatorname{tp}(a c)$. We know that $a \downarrow_{\emptyset} x_{n}$. By Stationarity, $\operatorname{tp}\left(x_{n} / a\right)=\operatorname{tp}(b / a)$, hence there exists an automorphism $f$ of $\mathbb{F}$ which fixes $a$ and maps $x_{n}$ to $b$. By Invariance, $f(a), f\left(x_{1}\right), \ldots, f\left(x_{n}\right)$ has the desired properties.

Lemma 2.3. Let $v_{1}, \ldots, v_{k}$ and $w_{1}, \ldots, w_{k}$ be geodesic sequences of vertices of $\mathbb{F}$ such that for every $1 \leq i<k$ we have $\operatorname{tp}\left(v_{i} v_{i+1}\right)=\operatorname{tp}\left(w_{i} w_{i+1}\right)$. Then $\operatorname{tp}\left(v_{1} \cdots v_{k}\right)=$ $\operatorname{tp}\left(w_{1} \cdots w_{k}\right)$.

Proof. We shall prove by induction on $m$ that $\operatorname{tp}\left(v_{1} \cdots v_{m}\right)=\operatorname{tp}\left(w_{1} \cdots w_{m}\right)$. For $m=2$ this is true by the assumption. Assume now that the statement is true for some $m$. Using the fact that $v_{1}, \ldots, v_{k}$ and $w_{1}, \ldots, w_{k}$ are geodesic sequences and Triviality we get that $v_{1} \cdots v_{m-1} \downarrow_{v_{m}} v_{m+1}$ and $w_{1} \cdots w_{m-1} \downarrow_{w_{m}} w_{m+1}$. By the assumption we have $\operatorname{tp}\left(v_{m} v_{m+1}\right)=\operatorname{tp}\left(w_{m} w_{i+m}\right)$, hence Stationarity together with Invariance and the induction hypothesis give $\operatorname{tp}\left(v_{1} \cdots v_{m+1}\right)=\operatorname{tp}\left(w_{1} \cdots w_{m+1}\right)$.

Proposition 2.4. Let $a, b, c$ be vertices of $\mathbb{F}$ satisfying the following:

(1) $a \downarrow_{b} c$,

(2) there is a geodesic sequence $a=v_{1}, \ldots, v_{k}=b$,

(3) there is a geodesic sequence $b=w_{1}, \ldots, w_{\ell}=c$.

Then there is a geodesic sequence $a=x_{1}, \ldots, x_{k+\ell-1}=c$ such that $\operatorname{tp}\left(x_{1} \cdots x_{k}\right)=$ $\operatorname{tp}\left(v_{1} \cdots v_{k}\right)$ and $\operatorname{tp}\left(x_{k} \cdots x_{k+\ell-1}\right)=\operatorname{tp}\left(w_{1} \cdots w_{\ell}\right)$.

Proof. Use Lemma 2.1 and the fact that all vertices have the same type $\ell-1$ times to extend $v_{1}, \ldots, v_{k}$ by vertices $w_{2}^{\prime}, \ldots, w_{\ell}^{\prime}$ such that $v_{1}, \ldots, v_{k}, w_{2}^{\prime}, \ldots, w_{\ell}^{\prime}$ is a geodesic sequence and for every $1 \leq i<\ell$ we have $\operatorname{tp}\left(w_{i}^{\prime} w_{i+1}^{\prime}\right)=\operatorname{tp}\left(w_{i} w_{i+1}\right)$, where we put $w_{1}^{\prime}=v_{k}$ to simplify the notation.

In particular, $w_{1}^{\prime}, \ldots, w_{\ell}^{\prime}$ is a geodesic sequence. Using Lemma 2.3 we get that $\operatorname{tp}\left(w_{1} \cdots w_{\ell}\right)=\operatorname{tp}\left(w_{1}^{\prime} \cdots w_{\ell}^{\prime}\right)$, so in particular $\operatorname{tp}\left(w_{1} w_{\ell}\right)=\operatorname{tp}\left(w_{1}^{\prime} w_{\ell}^{\prime}\right)$. Since $w_{1}=w_{1}^{\prime}=v_{k}$, we have that $\operatorname{tp}\left(w_{\ell} / v_{k}\right)=\operatorname{tp}\left(w_{\ell}^{\prime} / v_{k}\right)$. By the hypothesis and the construction, $w_{\ell} \downarrow_{v_{k}} v_{1}$ and $w_{\ell}^{\prime} \downarrow_{v_{k}} v_{1}$. Stationarity implies that $w_{\ell}^{\prime} \models \operatorname{tp}\left(w_{\ell} / v_{1} v_{k}\right)$, so in particular $w_{\ell}^{\prime} \models \operatorname{tp}\left(w_{\ell} / v_{1}\right)$.

In other words, there is an automorphism $g$ of $\mathbb{F}$ such that $g\left(v_{1}\right)=v_{1}$ and $g\left(w_{\ell}^{\prime}\right)=w_{\ell}$. The image of $v_{1}, \ldots, v_{k}, w_{2}^{\prime}, \ldots, w_{\ell}^{\prime}$ under $g$ then gives the desired geodesic sequence $x_{1}, \ldots, x_{k+\ell-1}$.

Let $a, b \in \mathbb{F}$ be distinct. We say that $b$ is almost free from $a$ if $a \bigsqcup_{\emptyset} b$ and for every $c \in \mathbb{F}$ different from $a, b$ such that $a \downarrow_{b} c$ it holds that $a \downarrow_{\emptyset} c$.

Observation 2.5. Let $a, b \in \mathbb{F}$ be such that $b$ is almost free from a. For every $a^{\prime}, b^{\prime} \in \mathbb{F}$ such that $\operatorname{tp}\left(a^{\prime} b^{\prime}\right)=\operatorname{tp}(a b)$ it holds that $b^{\prime}$ is almost free from $a^{\prime}$.

Lemma 2.6. Suppose that $\downarrow$ is bounded. For every $a \in \mathbb{F}$ and every finite $X \subseteq \mathbb{F}$ such that $a \notin X$ there is $b \in \mathbb{F}$ such that $a$ is almost free from $b, b$ is almost free from $a$, and $b \downarrow_{a} X$. In particular, $b \Perp_{\emptyset} a$ and $b \downarrow_{\emptyset} X$. 
Proof. We claim that there exist $a^{\prime}, b^{\prime} \in \mathbb{F}$ such that $b^{\prime}$ is almost free from $a^{\prime}$ and $a^{\prime}$ is almost free from $b^{\prime}$. Suppose that this is true. Since $\mathbb{F}$ is transitive, there is an automorphism $f$ such that $f\left(a^{\prime}\right)=a$. Pick $b \models \operatorname{tp}\left(f\left(b^{\prime}\right) / a\right)$ such that $b \downarrow_{a} X$. By Observation 2.5, $b$ is almost free from $a$ and $a$ is almost free from $b$. The "in particular" part is immediate using Corollary 1.10.

Hence it suffices to prove the claim. Pick $a^{\prime}, b^{\prime} \in \mathbb{F}$ such that $b^{\prime} \mathbb{L}_{\emptyset} a^{\prime}$ and the length of the longest geodesic sequence starting at $a^{\prime}$ finishing at $b^{\prime}$ is as large as possible. (As $\downarrow$ is bounded, such $a^{\prime}, b^{\prime}$ exist.) Pick $c \in \mathbb{F}$ such that $a^{\prime} \downarrow_{b^{\prime}} c$. By Proposition 2.4, we can extend the geodesic sequence from $a^{\prime}$ to $b^{\prime}$ by some $c^{\prime} \models \operatorname{tp}\left(c / b^{\prime}\right)$. By the properties of $a^{\prime}, b^{\prime}$ we get that $a^{\prime} \downarrow_{\emptyset} c^{\prime}$. Invariance and Stationarity then imply that $a^{\prime} \downarrow_{\emptyset} c$ and consequently $b^{\prime}$ is almost free from $a^{\prime}$.

To prove that $a^{\prime}$ is almost free from $b^{\prime}$, pick $c \in \mathbb{F}$ such that $b^{\prime} \downarrow_{a^{\prime}} c$. Since the reverse of a geodesic sequence is a geodesic sequence, we extend the geodesic sequence from $b^{\prime}$ to $a^{\prime}$ by some $c^{\prime} \models \operatorname{tp}\left(c / a^{\prime}\right)$ as above. Suppose that $b^{\prime} £_{\emptyset} c^{\prime}$. Since $\mathbb{F}$ is transitive, there is an automorphism $f$ such that $f\left(b^{\prime}\right)=a^{\prime}$. The image of the geodesic sequence from $b^{\prime}$ to $c^{\prime}$ is then a geodesic sequence starting at $a^{\prime}$ which is longer than the geodesic sequence from $a^{\prime}$ to $b^{\prime}$ we started with. This is a contradiction, hence $b^{\prime} \downarrow_{\emptyset} c^{\prime}$. As before, we get that $a^{\prime}$ is almost free from $b^{\prime}$ which concludes the proof.

\section{Proof of Theorem 1.1}

We will closely follow the proof from the Tent-Ziegler paper on the Urysohn sphere [TZ13a] and use the following result by Tent and Ziegler [TZ13b].

Definition 3.1. Let $\mathbb{F}$ be a countable structure with a stationary independence relation $\downarrow$, let $g \in \operatorname{Aut}(\mathbb{F})$, let $A \subseteq \mathbb{F}$ be finite and let $p=\operatorname{tp}(a / A)$ be a type. We say that $g$ moves $p$ almost maximally if there is a realisation $x \models p$ such that

$$
x \underset{A}{\downarrow} g(x) .
$$

Theorem 3.2 (Corollary 5.4, [TZ13b]). Let $\mathbb{F}$ be a countable structure with a stationary independence relation and let $g$ be an automorphism of $\mathbb{F}$ which moves every type over every finite set almost maximally. Then every element of $\operatorname{Aut}(\mathbb{F})$ is a product of sixteen conjugates of $g$.

Throughout the section, we fix $\mathbb{F}$ and $\downarrow$ as in Theorem 1.1 ( $\mathbb{F}$ is a transitive countable relational structure with a bounded 1-supported metric-like stationary independence relation $\downarrow$ ) and put $G=\operatorname{Aut}(\mathbb{F})$. As before, we may assume that $\mathbb{F}$ is homogeneous (this will slightly simplify the proof of Lemma 3.6).

Lemma 3.3. If $g \in G$ is not the identity then there is $a \in \mathbb{F}$ and $h \in G$ which is a product of $\|\downarrow\|$ conjugates of $g$ such that $a \downarrow_{\emptyset} h(a)$.

Proof. Let $a \in \mathbb{F}$ be such that $a \neq g(a)$ and pick $b \in \mathbb{F}$ such that $a \downarrow_{\emptyset} b$ (Existence). Use Lemma 2.2 to obtain a geodesic sequence $a=a_{0}, \ldots, a_{n}=b$ such that $n=$ $\|\downarrow\|$ and for every $0 \leq i \leq n-1$ we have $\operatorname{tp}\left(a_{i} a_{i+1}\right)=\operatorname{tp}(\operatorname{ag}(a))$. This means that there are automorphisms $h_{0}, \ldots, h_{n-1}$ such that $h_{i}(a)=a_{i}$ and $h_{i}(g(a))=a_{i+1}$. Then $h_{i} g h_{i}^{-1}$ moves $a_{i}$ to $a_{i+1}$ and the statement follows.

Lemma 3.4. Let $g \in G$ be such that for some $a \in \mathbb{F}$ we have $a \downarrow_{\emptyset} g(a)$. Then for every finite set $A \subset \mathbb{F}$ there is $x \in \mathbb{F}$ with $x \downarrow_{\emptyset} A$ and $x \neq g(x)$.

Proof. We may assume that $a \in A$. Put $Y=A \cup g^{-1}(A)$ and choose $b \in \mathbb{F}$ with $b \neq a$ and $b \bigsqcup_{\emptyset} a$ ( $\downarrow$ is metric-like) such that moreover $b \downarrow_{a} Y$ (Existence and Invariance). This means that $b \notin g^{-1}(A)$ (if $b \in g^{-1}(A)$, then $b \in Y$, so $b \downarrow_{a} b$, 
which is in contradiction with part (1) of Definition 1.8) and hence $g(b) \notin A$. We know that $a \downarrow_{\emptyset} g^{-1}(a)$ (by Invariance) and also $b \downarrow_{a} g^{-1}(a)$, thus $b \downarrow_{\emptyset} g^{-1}(a)$ (Transitivity) and so $g(b) \downarrow_{\emptyset} a$ (Invariance). This means that $b \neq g(b)$ and therefore $g(b) \notin A \cup\{b\}$.

Use Lemma 2.6 to obtain $x \in \mathbb{F}$ such that $x \mathbb{\ell}_{\emptyset} g(b)$ and $x \downarrow_{\emptyset} A b$. By Monotonicity, $x \downarrow_{\emptyset} A$ and $x \downarrow_{\emptyset} b$, hence also $g(x) \downarrow_{\emptyset} g(b)$, thus $x \neq g(x)$.

Let $X \subset \mathbb{F}$ be a finite set and let $a \in \mathbb{F}$ be such that $a \downarrow_{\emptyset} X$. We call the type $\operatorname{tp}(a / X)$ a free type. (It is the unique such type over $X$.)

Lemma 3.5. Let $g \in G$ be such that for every free type $p$ there is a realisation $a \models p$ with $g(a) \neq a$. Then for every finite $X \subset \mathbb{F}$ and every type $q=\operatorname{tp}(x / X)$ with $x \notin X$, there is a realisation $c \models q$ such that $g(c) \neq c$.

Proof. Let $a$ be a vertex such that $a \downarrow_{\emptyset} X$ and $g(a) \neq a$ ( $a$ exists by the assumptions of this lemma) and let $b \models q$ be such that $b \downarrow_{X} g(a)$.

If $b \mathbb{\ell}_{\emptyset} g(a)$ then pick $c \models q$ such that $c \downarrow_{X} a g(a)$. This means that $c \mathbb{\ell}_{\emptyset} g(a)$ (by Stationarity and Invariance) and $c \downarrow_{\emptyset} a$ (by Transitivity), giving us $g(c) \neq c$.

So we have $b \downarrow_{\emptyset} g(a)$. Use Lemma 2.6 to obtain $a^{\prime} \in \mathbb{F}$ such that $a^{\prime} \mathbb{L}_{\emptyset} b$, $a^{\prime} \downarrow_{\emptyset} X$, and $a^{\prime}$ is almost free from $b$. By Stationarity, we have that $a \models \operatorname{tp}\left(a^{\prime} / X\right)$, hence there is $f \in G$ fixing $X$ pointwise such that $f\left(a^{\prime}\right)=a$. Put $c^{\prime}=f(b)$. In particular, $c^{\prime} \models q, a \chi_{\emptyset} c^{\prime}$, and $a$ is almost free from $c^{\prime}$ (Observation 2.5).

Choose $c \models \operatorname{tp}\left(c^{\prime} / X a\right)$ such that $c \downarrow_{X a} g(a)$. In particular, $c \mathbb{\ell}_{\emptyset} a$ (Invariance). By Observation 2.5, $a$ is almost free from $c$. Using 1-supportedness, $c \downarrow_{X a} g(a)$ implies that either $c \downarrow_{a} g(a)$ (in which case $c \downarrow_{\emptyset} g(a)$ and hence $g(c) \neq c$ ), or $c \downarrow_{X} g(a)$. In this case we know that $\operatorname{tp}(c / X)=\operatorname{tp}(b / X)$ and $b \downarrow_{X} g(a)$ (using Perfect triviality on $\left.b \downarrow_{\emptyset} g(a)\right)$, hence by Stationarity and Invariance, $c \downarrow_{\emptyset} g(a)$, thus again $g(c) \neq c$.

We say that $g \in G$ moves type $p$ by distance $k$ if there is $a \models p$ and a geodesic sequence $a=a_{0}, \ldots, a_{k}=g(a)$. If $p=\operatorname{tp}(x / X)$ is a type and $h$ is an automorphism or a partial automorphism defined on a finite set such that $X \subseteq \operatorname{Dom}(h)$, we denote $h(p)=\operatorname{tp}\left(h^{\prime}(x) / h^{\prime}(X)\right)$, where $h^{\prime}$ is some automorphism of $\mathbb{F}$ extending $h$ (remember that we assumed that $\mathbb{F}$ is homogeneous).

Lemma 3.6. Let $g \in G$ be such that $g$ moves all types almost maximally or by distance $n$. Then there exists $h \in G$ such that $[g, h]=g^{-1} h^{-1} g h$ moves all types almost maximally or by distance $2 n$.

Proof. As in [TZ13a], we construct $h$ by a "back-and-forth" construction as the union of a chain of finite partial automorphisms. We show the following: Let $h^{\prime}$ be already defined on a finite set $U$ and let $p=\operatorname{tp}(x / X)$ be a type. Then $h^{\prime}$ has an extension $h$ such that $[g, h]$ moves $p$ almost maximally or by distance $2 n$.

We can assume that $X \cup g^{-1}(X) \subseteq U$. Put $V=h^{\prime}(U)$. Let $a^{\prime}$ be a realisation of $p$ such that $a^{\prime} \downarrow_{X} U g^{-1}(U)$ and let $b^{\prime}$ be a realisation of $h^{\prime}\left(\operatorname{tp}\left(a^{\prime} / U\right)\right.$ ) (which is a type over $V)$. By the hypothesis on $g$ there are realisations $a \models \operatorname{tp}\left(a^{\prime} / U g^{-1}(U)\right)$ and $b \models \operatorname{tp}\left(b^{\prime} / V\right)$ such that either $a \downarrow_{U g^{-1}(U)} g(a)$, or there is a geodesic sequence $a=a_{0}, \ldots, a_{n}=g(a)$ and similarly for $b$. We also have

$$
a \underset{X}{\downarrow} U g^{-1}(U) \text { and } b \underset{h^{\prime}(X)}{\downarrow} V .
$$

Let $h_{0}$ be the isomorphism $U a \simeq V b$ and let $c$ be a realisation of $h_{0}^{-1}(\operatorname{tp}(g(b) / V b))$ (which is a type over $U a$ ) such that $c \downarrow_{U a} g(a)$. Put $h$ to be the isomorphism 
$U a c \simeq V b g(b)$. Observe that $[g, h](a)=g^{-1}(c)$. It remains to prove that $a$ witnesses that $[g, h]$ moves $p$ almost maximally or by distance $2 n$.

Since $a \downarrow_{X} g^{-1}(U)$, we know that $g(a) \downarrow_{g(X)} U$. Using Metricity, we get

$$
c \underset{g(X) a}{\perp} g(a)
$$

thus from 1-supportedness we know that either $c \downarrow_{a} g(a)$ or $c \downarrow_{g(X)} g(a)$. In the second case we get $g^{-1}(c) \downarrow_{X} a$, which implies that $[g, h]$ moves $p$ almost maximally. Hence we can assume that

$$
c \underset{a}{\downarrow} g(a) .
$$

By the choice of $a$ and $b$ we know that one of the following cases occurs:

(1) First suppose that there are geodesic sequences $b=b_{0}, \ldots, b_{n}=g(b)$ and $g(a)=a_{0}, \ldots, a_{n}=a$ (the reverse of a geodesic sequence is a geodesic sequence by Symmetry). From the construction we know that $\operatorname{tp}(a c)=$ $\operatorname{tp}(b g(b))$. This implies that there is a geodesic sequence $a=c_{0}, \ldots, c_{n}=c$. Since $g(a) \downarrow_{a} c$, Proposition 2.4 gives a geodesic sequence starting at $g(a)$ and finishing at $c$ using $2 n+1$ vertices (including $c$ and $g(a)$ ). Finally, taking the image of this sequence under $g^{-1}$ gives a geodesic sequence starting at $a$ and finishing at $g^{-1}(c)=[g, h](a)$ using $2 n+1$ vertices. This means that $a$ witnesses that $[g, h]$ moves $p$ by distance $2 n$.

(2) Now assume that $a \downarrow_{U g^{-1}(U)} g(a)$. Then in fact we have $a \downarrow_{X} g(a)$, because $a \downarrow_{X} U g^{-1}(U)$ (Metricity). As $U \supseteq X g^{-1}(X), a \downarrow_{X} U$ also implies $g(a) \perp_{g(X)} X$ (by Invariance and Monotonicity), which together with $a \downarrow_{X} g(a)$ implies $a \downarrow_{g(X)} g(a)$ (Metricity). Thus from $c \downarrow_{a} g(a)$ we get $c \downarrow_{g(X)} g(a)$ (yet again Metricity) and thus $g^{-1}(c) \downarrow_{X} a$, i.e. $a$ witnesses that $[g, h]$ moves $p$ almost maximally.

(3) Otherwise we have $b \downarrow_{V} g(b)$. Using that $h$ is an isomorphism of $U a c$ and $V b g(b)$ and Invariance we obtain $a \downarrow_{U} c$. Then we get $a \downarrow_{X} c$, because $a \downarrow_{X} U$ (Metricity), and then, combining with $c \downarrow_{a} g(a)$ using Metricity again, we obtain $c \downarrow_{X} g(a)$. As in the previous case, $a \downarrow_{X} U$ implies $g(a) \downarrow_{g(X)} X$ and hence $c \downarrow_{g(X)} g(a)$, or $g^{-1}(c) \downarrow_{X} a$, i.e. $a$ witnesses that $[g, h]$ moves $p$ almost maximally.

Now we prove the following proposition, Theorem 1.1 is then its direct consequence.

Proposition 3.7. Let $\mathbb{F}$ be a countable relational structure with a bounded 1supported metric-like stationary independence relation $\downarrow$ and let $g$ be a non-identity automorphism of $\mathbb{F}$. Then there is an automorphism of $\mathbb{F}$ which is a product of at most $2\|\downarrow\|^{2}$ conjugates of $g$ and $g^{-1}$ and moves every type over every finite set almost maximally.

Proof. From Lemma 3.3 we get an automorphism $g_{0}$ which is a product of at most $\|\downarrow\|$ conjugates of $g$ such that there is $a \in \mathbb{F}$ with $a \downarrow_{\emptyset} g_{0}(a)$. Using Lemma 3.4 we get that in fact for every free type there is a realisation which is not fixed by $g_{0}$.

Let $p=\operatorname{tp}(x / X)$ be a type. Either $x \in X$ (then $x \downarrow_{X} g(x)$, hence $g_{0}$ moves $q$ almost maximally), or $x \notin X$ and thus by Lemma 3.5 there is a realisation of $p$ which is not fixed by $g_{0}$. This means that $g_{0}$ moves all types almost maximally or by distance 1 . 
Put $n=\left\lceil\log _{2}(\|\downarrow\|)\right\rceil$ and construct a sequence $g_{0}, g_{1}, \ldots, g_{n}$ of automorphisms of $\mathbb{F}$ using Lemma 3.6 such that every $g_{i}$ moves all types almost maximally or by distance $2^{i}$, and if $i \geq 1$ then $g_{i}$ is a product of two conjugates of $g_{i-1}$ and $g_{i-1}^{-1}$. For $g_{n}$ we get that it moves every type almost maximally or by distance at least $\|\downarrow\|$. In the latter case, we have for every type $p$ a realisation $a \models p$ and a geodesic sequence $a=a_{0}, \ldots, a_{k}=g(a)$, where $k \geq\|\downarrow\|$. Boundedness (Definition 1.12) implies that $a \downarrow_{\emptyset} g(a)$, i.e. $g_{n}$ moves $p$ almost maximally, and hence $g_{n}$ moves all types almost maximally.

By the construction, $g_{n}$ is a product of at most $2^{\left\lceil\log _{2}(\|\downarrow\|)\right\rceil}$ conjugates of $g_{0}$ and $g_{0}^{-1}$, hence a product of at most $2^{\left\lceil\log _{2}(\|\downarrow\|)\right\rceil}\|\downarrow\| \leq 2\|\downarrow\|^{2}$ conjugates of $g$ and $g^{-1}$.

Proof of Theorem 1.1. Let $g$ be a non-identity automorphism of $\mathbb{F}$. We need to prove that if $N$ is a normal subgroup of $G$ such that $g \in N$, then $N=G$. If $g \in N$, then clearly $g^{-1} \in N$. Let $h \in G$. By Proposition 3.7 and Theorem 3.2, we know that $h$ can be written as a product of conjugates of $g$ and $g^{-1}$, hence $h \in N$. This is true for every $h \in G$, hence $N=G$ and $G$ is simple.

\section{Corollaries}

In this section we prove Theorems 1.2 and 1.3 .

4.1. Semigroup-valued metric spaces. We say that a tuple $\mathfrak{M}=(M, \oplus, \preceq)$ is a partially ordered commutative semigroup if the following hold:

(1) $(M, \oplus)$ is a commutative semigroup,

(2) $(M, \preceq)$ is a partial order which is reflexive $(a \preceq a$ for every $a \in M)$,

(3) for every $a, b \in M$ it holds that $a \preceq a \oplus b$, and

(4) for every $a, b, c \in M$ it holds that if $b \preceq c$ then $a \oplus b \preceq a \oplus c$ ( $\oplus$ is monotone with respect to $\preceq$ ).

$\mathfrak{M}$ is archimedean if for every $a, b \in \mathfrak{M}$ there is an integer $n$ such that $n \times a \succeq b$, where by $n \times a$ we mean

$$
\underbrace{a \oplus a \oplus \cdots \oplus a}_{n \text { times }}
$$

Note that if $\mathfrak{M}$ is archimedean and non-trivial, it follows that $\mathfrak{M}$ does not have an identity.

Let $L$ be a set. An $L$-edge-labelled graph is a tuple $\mathbf{A}=(A, E, d)$, where $E \subseteq\left(\begin{array}{c}A \\ 2\end{array}\right)$ and $d$ is a function $E \rightarrow L$. Clearly, the set $E$ can be inferred from the function $d$ and thus we omit it. For simplicity, we write $d(x, y)$ instead of $d(\{x, y\})$ and we put $d(x, x)=0$, where 0 is a symbol which is not an element of $\mathfrak{M}$. When convenient, we naturally understand 0 as the neutral element with respect to $\oplus$ and as the minimum element of $\preceq$.

We say that $\mathbf{A}$ is complete if the graph $(A, E)$ is a complete graph. Note that an $L$-edge-labelled graph can equivalently be viewed as a relational structure with an irreflexive binary symmetric relation $R^{m}$ for every $m \in L$ such that every pair of vertices is in at most one relation.

For a partially ordered commutative semigroup $\mathfrak{M}=(M, \oplus, \preceq)$, a complete $\mathfrak{M}$ edge-labelled graph $\mathbf{A}=(A, d)$ is an $\mathfrak{M}$-metric space if for every triple $a, b, c \in A$ of distinct vertices it holds that $d(a, b) \preceq d(a, c) \oplus d(b, c)$ (the triangle inequality).

Let $\mathbb{F}$ be an $\mathfrak{M}$-metric space. We say that $\mathbb{F}$ admits an $\mathfrak{M}$-shortest path independence relation if for every $a, b \in \mathbb{F}$ and $C \subseteq \mathbb{F}$ finite we have that $\{d(a, c) \oplus d(c, b)$ : $c \in C\}$ has an infimum with respect to $\preceq$ (note that $C$ can be empty which implies

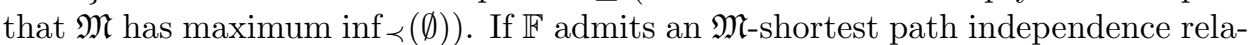
tion, then its $\mathfrak{M}$-shortest path independence relation is a ternary relation $\downarrow$ defined 
on finite subsets of $\mathbb{F}$ by putting $A \downarrow_{C} B$ if and only if for every $a \in A$ and every $b \in B$ it holds that $d(a, b)=\inf _{\preceq}\{d(a, c) \oplus d(c, b): c \in C\}$.

Generalising concepts of Sauer [Sau12], Conant [Con19] (see also [HKN17]) and Braunfeld [Bra17] (see also [KPR18]), Hubička, Konečný and Nešetřil [Kon19, HKN18] introduced the framework of semigroup-valued metric spaces, which served as a motivation for this paper. Given a partially ordered commutative semigroup $\mathfrak{M}=(M, \oplus, \preceq)$ and a "nice" family $\mathcal{F}$ of $\mathfrak{M}$-edge-labelled cycles, the structures of interest are $\mathfrak{M}$-metric spaces which moreover contain no homomorphic images of members of $\mathcal{F}$. We will denote the class of all such finite structures $\mathcal{M}_{\mathfrak{M}}^{\mathcal{F}}$.

The conditions of $\mathcal{F}$ are strong enough that one can then prove that $\mathcal{M}_{\mathfrak{M}}^{\mathcal{F}}$ is a strong amalgamation class, its Fraïssé limit admits an $\mathfrak{M}$-shortest path independence relation which is a SIR (provided that $\mathfrak{M}$ has a maximum, otherwise one can still get a local SIR), it has EPPA (for background, see [HKN19, Sin17]) and a precompact Ramsey expansion (for background, see [HN19, NVT15]), but they are general enough that most known binary symmetric homogeneous structures can be viewed as such a semigroup-valued metric space. In fact, it is conjectured that every primitive transitive homogeneous structure in a finite binary symmetric language with trivial algebraic closures admits such an interpretation (Conjecture 1 in $[$ Kon19]).

Now we are ready to prove Theorem 1.2.

Proof of Theorem 1.2. We need to prove that $\downarrow$ is metric-like and bounded. (In fact, we do not need 1-supportedness for this, we only need it later in order to apply Theorem 1.1.)

Since $\mathbb{F}$ is homogeneous, all vertices have the same type. As $d(x, y)=0$ if and only if $x=y$ and $\oplus$ is monotone with respect to $\preceq$, it follows that if $a \notin A$, then $a \mathbb{X}_{A} a$. The fact that there are $a \neq b \in \mathbb{F}$ such that $a \mathbb{X}_{\emptyset} b$ follows from Stationarity, the fact that $\mathfrak{M}$ has at least two elements (remember that $0 \notin \mathfrak{M}$ ) and the fact that $\mathbb{F}$ realises all distances.

Suppose now that $a \downarrow_{C} b$. If there was $c^{\prime} \in \mathbb{F} \backslash C$ such that $a \downarrow_{C c^{\prime}} b$, this would mean that $\inf _{\preceq}\left\{d(a, c) \oplus d(c, b): c \in C \cup\left\{c^{\prime}\right\}\right\} \prec\{d(a, c) \oplus d(c, b): c \in C\}=d(a, b)$, hence $d\left(a, c^{\prime}\right) \oplus d\left(c^{\prime}, b\right) \nsucceq d(a, b)$, in other words, $a b c^{\prime}$ violates the triangle inequality which is a contradiction. Consequently, $\downarrow$ satisfies Perfect triviality and hence $\downarrow$ is metric-like.

Next we prove that $\downarrow$ is bounded. Denote by 1 the maximum element of $\mathfrak{M}$ ( $\mathfrak{M}$ is finite and hence there is such an element). Assume that there are $a, b \in \mathfrak{M}$ such that $a \oplus b=a$. This means (by associativity) that $a \oplus(n \times b)=a$ for every $n$. Let $c \in \mathfrak{M}$ be arbitrary. By archimedeanity there is $n$ such that $n \times b \succeq c$. But then $a=a \oplus(n \times b) \succeq c$. Hence $a \succeq c$ for every $c \in \mathfrak{M}$, that is, $a=1$. In other words, for every $a, b \in \mathfrak{M} \backslash\{1\}$ it holds that $a \oplus b \succ a$, which implies that whenever $a_{1}, \ldots, a_{|\mathfrak{M}|} \in \mathfrak{M}$, then

$$
\bigoplus_{i=1}^{|\mathfrak{M}|} a_{i}=1 .
$$

We can use this observation to prove that $\|\downarrow\| \leq|\mathfrak{M}|$. Indeed, if $a_{0}, \ldots, a_{|\mathfrak{M}|}$ is a geodesic sequence, we know that $d\left(a_{0}, a_{i+1}\right)=d\left(a_{0}, a_{i}\right) \oplus d\left(a_{i}, a_{i+1}\right)$. Using induction we get that

$$
d\left(a_{0}, a_{|\mathfrak{M}|}\right)=d\left(a_{1}, a_{2}\right) \oplus d\left(a_{2}, a_{3}\right) \oplus \cdots \oplus d\left(a_{|\mathfrak{M}|-1}, a_{|\mathfrak{M}|}\right),
$$

that is, $d\left(a_{0}, a_{|\mathfrak{M}|}\right)$ is a sum of $|\mathfrak{M}|$ elements of $\mathfrak{M}$ and hence $d\left(a_{0}, a_{|\mathfrak{M}|}\right)=1$, which means that indeed $a_{0} \downarrow_{\emptyset} a_{|\mathfrak{M}|}$. 
We have proved that $\downarrow$ is bounded and metric-like, hence we can apply Theorem 1.1 to show that $\operatorname{Aut}(\mathbb{F})$ is simple.

Note that whenever $\preceq$ is a linear order, the corresponding $\mathfrak{M}$-shortest path independence relation is necessarily 1 -supported. The following theorem is a direct consequence of this fact, Theorem 1.2 and existing results on semigroup-valued metric spaces [Kon19, HKN18].

Let $S \subseteq \mathbb{R}^{+}$be a finite subset of positive reals such that the following operation $\oplus_{S}: S^{2} \rightarrow S$ is associative:

$$
a \oplus_{S} b=\max \{x \in S: x \leq a+b\} .
$$

Delhommé, Laflamme, Pouzet, and Sauer [DLPS07] studied and Sauer later classified [Sau13a, Sau13b] such subsets. Ramsey expansions for all such classes of $\left(S, \oplus_{S}, \leq\right)$-metric spaces were obtained by Hubička and Nešetřil [HN19], and Hubička, Konečný, Nešetřil and Sauer [HKNS20] (Nguyen Van Thé [NVT09] earlier proved some partial results). We contribute to the study of such classes by the following result:

Theorem 4.1. Let $S \subseteq \mathbb{R}^{+}$be a finite subset of positive reals such that $\mathfrak{M}_{S}=$ $\left(S, \oplus_{S}, \leq\right)$ is an archimedean partially ordered commutative semigroup. Then the automorphism group of the Fraïsé limit of the class of all finite $\mathfrak{M}_{S}$-metric spaces is simple.

4.2. Metrically homogeneous graphs. A metrically homogeneous graph is a graph whose path-metric is a homogeneous metric space. Cherlin [Che11, Che17] gave a list of such graphs by describing the corresponding amalgamation classes of metric spaces. The vast majority of the list is occupied by the 5 -parameter classes $\mathcal{A}_{K_{1}, K_{2}, C_{1}, C_{2}}^{\delta}$, where $\delta$ denotes the diameter of such spaces (i.e. they only use distances $\{1, \ldots, \delta\}$ ) and the other four parameters describe four different families of forbidden triangles (for example, all triangles of odd perimeter smaller than $2 K_{1}$ are forbidden).

Aranda, Bradley-Williams, Hubička, Karamanlis, Kompatscher, Konečný and Pawliuk $\left[\mathrm{ABWH}^{+} 17 \mathrm{c}, \mathrm{ABWH}^{+} 17 \mathrm{a}, \mathrm{ABWH}^{+} 17 \mathrm{~b}\right]$ studied EPPA, Ramsey expansions and (local) SIR's for these classes (see also [Kon18, EHKN20, Kon20]). In particular, if $\mathcal{A}_{K_{1}, K_{2}, C_{1}, C_{2}}^{\delta}$ is primitive (i.e. it is neither antipodal nor bipartite) and $\delta$ is finite, it can be shown using another result of Hubička, Kompatscher and Konečný [HKK18] that these (local) stationary independence relations are 1supported and can be viewed as $\mathfrak{M}$-shortest path independence relations [Kon19] with a finite archimedean $\mathfrak{M}$, which means that Theorem 1.3 is a direct consequence of Theorem 1.2.

\section{CONCLUSiOn}

We conclude with two questions and a conjecture. The first question is a particular instance of the general question whether 1-supportedness is necessary.

Question 5.1. Consider the structure $\mathbb{M}_{k}$ from Example 3, that is, the Fraïssé limit of all finite $[n]^{k}$-metric spaces (which are in fact semigroup-valued metric spaces in the sense of Section 4.1). Is the automorphism group of $\mathbb{M}_{k}$ simple? (For $k \geq 2$ and $n$ large enough - if, for example, $n=3$, it is in fact a free amalgamation class, as $(2, \ldots, 2)$ is a free relation.)

The obvious next step is to generalise our results to countable archimedean semigroups which do not have to contain a maximum element, thereby obtaining and analogue of Tent and Ziegler's result on the Urysohn space [TZ13b]. We believe that such a generalisation is quite straightforward. However, there are structures 
in infinite language which do not even admit a SIR, although they are also very much metric-like. One example is the sharp Urysohn space:

Question 5.2. Let $\mathbb{U}^{\#}$ be the Fraïssé limit of the class of all finite complete $\mathbb{Q}^{+}$edge-labelled graphs (here $\mathbb{Q}^{+}$is the set of all positive rational numbers) which contain no triangles $a, b, c$ with $d(a, b) \geq d(a, c)+d(b, c)$ (that is, the triangle inequality is sharp). Is the automorphism group of $\mathbb{U}^{\#}$ simple modulo bounded automorphisms?

Note that if we consider $\mathbb{N}$ instead of $\mathbb{Q}^{+}$, the resulting structure can be understood as an $\mathfrak{M}$-metric space (putting $a \oplus b=a+b-1$ and $a \preceq b$ if $a \leq b$ ).

Remark 5.3. The sharp Urysohn space is a very peculiar structure, because although it does not admit a SIR, it has EPPA, APA and it is Ramsey when equipped with a (free) linear order.

The following conjecture and question are closely related to a conjecture from [Kon19].

Conjecture 5.4. Every countable homogeneous complete L-edge-labelled graph with $2 \leq|L|<\infty$, primitive automorphism group and trivial algebraic closure admits a metric-like SIR.

Question 5.5. Assume that $\mathbb{F}$ is a transitive countable structure with a metric-like $S I R \downarrow$ such that $\operatorname{tp}(a b)=\operatorname{tp}(b a)$ for every $a, b \in \mathbb{F}$. Can one define a partially ordered commutative semigroup $\mathfrak{M}$ on the 2-types of $\mathbb{F}$ such that $\downarrow$ is the $\mathfrak{M}$ shortest path independence relation? If the answer is yes, is it true that for every $a \neq b \neq c \in \mathbb{F}$ it holds that $\operatorname{tp}(a b) \preceq \operatorname{tp}(a c) \oplus \operatorname{tp}(b c)$ ?

The obvious special cases of Question 5.5 are for finitely many 2-types, 1supported $\downarrow$, bounded $\downarrow$, and their combinations. It is not true that the conditions of Question 5.5 imply that the structure at hand is an $\mathfrak{M}$-metric space in the sense of [Kon19, HKN18]. For example, suppose that $\mathbb{F}$ is the Fraïssé limit of the class of all $[n]^{1}$-metric spaces which also contain a ternary relation $R$ such that if $(a, b, c) \in R$, then $d(a, b)=d(b, c)=d(c, a)=1$. The standard $([n],+, \leq)$-shortest path independence relation is the desired SIR on $\mathbb{F}$.

\section{REFERENCES}

$\left[\mathrm{ABWH}^{+} 17 \mathrm{a}\right]$ Andres Aranda, David Bradley-Williams, Eng Keat Hng, Jan Hubička, Miltiadis Karamanlis, Michael Kompatscher, Matěj Konečný, and Micheal Pawliuk. Completing graphs to metric spaces. Electronic Notes in Discrete Mathematics, 61:5360, 2017. The European Conference on Combinatorics, Graph Theory and Applications (EUROCOMB'17).

$\left[\mathrm{ABWH}^{+} 17 \mathrm{~b}\right]$ Andres Aranda, David Bradley-Williams, Eng Keat Hng, Jan Hubička, Miltiadis Karamanlis, Michael Kompatscher, Matěj Konečný, and Micheal Pawliuk. Completing graphs to metric spaces. arXiv:1706.00295, accepted to Contributions to Discrete Mathematics, 2017.

$\left[\mathrm{ABWH}^{+} 17 \mathrm{c}\right]$ Andres Aranda, David Bradley-Williams, Jan Hubička, Miltiadis Karamanlis, Michael Kompatscher, Matěj Konečný, and Micheal Pawliuk. Ramsey expansions of metrically homogeneous graphs. Accepted to European Journal of Combinatorics, arXiv:1707.02612, 2017.

[Bau16] Andreas Baudisch. Free amalgamation and automorphism groups. The Journal of Symbolic Logic, 81(3):936-947, 2016.

[Bra17] Samuel Braunfeld. Ramsey expansions of $\Lambda$-ultrametric spaces. arXiv:1710.01193, 2017.

[Che98] Gregory Cherlin. The Classification of Countable Homogeneous Directed Graphs and Countable Homogeneous $N$-tournaments. Number 621 in Memoirs of the American Mathematical Society. American Mathematical Society, 1998.

[Che11] Gregory Cherlin. Two problems on homogeneous structures, revisited. Model theoretic methods in finite combinatorics, 558:319-415, 2011. 
[Che17] Gregory Cherlin. Homogeneous ordered graphs and metrically homogeneous graphs. Submitted, December 2017.

[CKT20] F. Calderoni, A. Kwiatkowska, and K. Tent. Simplicity of the automorphism groups of order and tournament expansions of homogeneous structures. Preprint, arXiv:1808.05177, 2020.

[Con19] Gabriel Conant. Extending partial isometries of generalized metric spaces. Fundamenta Mathematicae, 244:1-16, 2019.

[DLPS07] Christian Delhommé, Claude Laflamme, Maurice Pouzet, and Norbert W. Sauer. Divisibility of countable metric spaces. European Journal of Combinatorics, 28(6):1746-1769, 2007.

[EGT16] David M. Evans, Zaniar Ghadernezhad, and Katrin Tent. Simplicity of the automorphism groups of some hrushovski constructions. Annals of Pure and Applied Logic, 167(1):22-48, 2016.

[EHKN20] David M. Evans, Jan Hubička, Matěj Konečný, and Jaroslav Nešetřil. EPPA for two-graphs and antipodal metric spaces. Proceedings of the American Mathematical Society, 148:1901-1915, 2020.

[HKK18] Jan Hubička, Michael Kompatscher, and Matěj Konečný. Forbidden cycles in metrically homogeneous graphs. Submitted, arXiv:1808.05177, 2018.

[HKN17] Jan Hubička, Matěj Konečný, and Jaroslav Nešetřil. Conant's generalised metric spaces are Ramsey. Accepted to Contributions to Discrete Mathematics, arXiv:1710.04690, 2017.

[HKN18] Jan Hubička, Matěj Konečný, and Jaroslav Nešetřil. Semigroup-valued metric spaces: Ramsey expansions and EPPA. In preparation, 2018.

[HKN19] Jan Hubička, Matěj Konečný, and Jaroslav Nešetřil. All those EPPA classes (strengthenings of the Herwig-Lascar theorem). Submitted, arXiv:1902.03855, 2019.

[HKNS20] Jan Hubička, Matěj Konečný, Jaroslav Nešetřil, and Norbert Sauer. Ramsey expansions and EPPA for $S$-metric spaces. To appear, 2020.

[HN19] Jan Hubička and Jaroslav Nešetřil. All those Ramsey classes (Ramsey classes with closures and forbidden homomorphisms). Advances in Mathematics, 356C:106791, 2019.

[Kon18] Matěj Konečný. Combinatorial properties of metrically homogeneous graphs. Bachelor's thesis, Charles University, 2018. arXiv:1805.07425.

[Kon19] Matěj Konečný. Semigroup-valued metric spaces. Master's thesis, Charles University, 2019. arXiv:1810.08963.

[Kon20] Matěj Konečný. Extending partial isometries of antipodal graphs. Discrete Mathematics, 343(1):111633, 2020.

[KPR18] Mustapha Kabil, Maurice Pouzet, and Ivo G. Rosenberg. Free monoids and generalized metric spaces. European Journal of Combinatorics, 2018.

[KS19] Itay Kaplan and Pierre Simon. Automorphism groups of finite topological rank. Transactions of the American Mathematical Society, 372(3):2011-2043, 2019.

[Li18] Yibei Li. Simplicity of the automorphism groups of some binary homogeneous structures determined by triangle constraints. arXiv:1806.01671, 2018.

[Li19] Yibei Li. Automorphism groups of homogeneous structures with stationary weak independence relations. arXiv:1911.08540, 2019.

[Li20] Yibei Li. Automorphism groups of linearly ordered homogeneous structures. arXiv:2009.02475, 2020.

[MT11] Dugald Macpherson and Katrin Tent. Simplicity of some automorphism groups. Journal of Algebra, 342(1):40-52, 2011.

[NVT09] Lionel Nguyen Van Thé. Ramsey degrees of finite ultrametric spaces, ultrametric Urysohn spaces and dynamics of their isometry groups. European Journal of Combinatorics, 30(4):934-945, 2009.

[NVT15] Lionel Nguyen Van Thé. A survey on structural Ramsey theory and topological dynamics with the Kechris-Pestov-Todorcevic correspondence in mind. Selected Topics in Combinatorial Analysis, 17(25):189-207, 2015.

[Sau12] Norbert W. Sauer. Vertex partitions of metric spaces with finite distance sets. Discrete Mathematics, 312(1):119-128, 2012.

[Sau13a] Norbert W. Sauer. Distance sets of Urysohn metric spaces. Canadian Journal of Mathematics, 65(1):222-240, 2013.

[Sau13b] Norbert W. Sauer. Oscillation of Urysohn type spaces. In Asymptotic geometric analysis, pages 247-270. Springer, 2013.

[Sin17] Daoud Siniora. Automorphism Groups of Homogeneous Structures. PhD thesis, University of Leeds, March 2017. 
[TZ13a] Katrin Tent and Martin Ziegler. The isometry group of the bounded urysohn space is simple. Bulletin of the London Mathematical Society, 45(5):1026-1030, 2013.

[TZ13b] Katrin Tent and Martin Ziegler. On the isometry group of the Urysohn space. Journal of the London Mathematical Society, 87(1):289-303, 2013.

Department of Mathematics, Imperial College London, London SW7 2AZ, UK.

Email address: david.evans@imperial.ac.uk

Department of Applied Mathematics (KAM), Charles University, Prague, Czech RePUBLIC

Email address: hubicka@kam.mff.cuni.cz

Department of Applied Mathematics (KAM), Charles University, Prague, Czech RePUBLIC

Email address: matej@kam.mff.cuni.cz

Imperial College London, London SW7 2AZ, UK.

Email address: yibei.li16@imperial.ac.uk

Mathematisches Institut, Albert-Ludwigs-Universität Freiburg, D-79104 Freiburg, GERMANY

Email address: ziegler@uni-freiburg.de 